\title{
Serious problem of oral health and dental evaluation before surgery
}

\author{
Silvay $\mathrm{G}^{1}$, Zafirova $\mathrm{Z}^{1}$, Desiderio $\mathrm{D}^{2}$, Valauri $\mathrm{D}^{3}$, Castillo $\mathrm{J}^{4}$ \\ Department of Anesthesiology, Icahn School of Medicine at Mount Sinai, New York, U.S.A. \\ George.Silvay@mountsinai.org
}

In 2008, my close friend, very active and physically fit businessman, was diagnosed with aortic stenosis and was scheduled for an elective aortic valve replacement. He was 67 years old, and until his recent diagnosis of severe aortic stenosis, he had no medical problems. He had been undergoing regular dental evaluations and treatments every six months. Perioperative routine infection prophylaxis with vancomycin and cefazolin was administered and he had an uneventful aortic valve replacement with a tissue valve. He was discharged from the hospital six days later.

Three weeks following the operation, he started to feel weak and his cardiologist noted fever; thorough examination resulted in diagnosis of bacterial endocarditis. Careful search for the initial source of inflammation pointed to a dental infection, which originated from the tooth's pulp following endodontic treatment (root canal). Following six months of intensive treatment of infection, he had significant prosthetic aortic regurgitation and underwent a second aortic valve replacement.

This case underscores the important value of oral health in cardiovascular surgery. In the present communication, we will discuss the current view on the risks and benefits of optimizing the dental health before high-risk surgery, including cardiothoracic and vascular surgeries, organ transplantations, knee/hip replacements, and complex oncological operations with immunosuppression therapy.

The old theories of the connection between oral and systemic health can be traced back to the 19th century (1). During the last decades, this relationship has been revisited in multiple publications. In 2000, the US surgeon general reported on oral health in the United States, highlighting it importance: "The mouth reflects general health and well-being. The oral cavity is a readily accessible and visible part of the body and provides the window to assess the overall medical status of the patient”. Former surgeon general

${ }^{1}$ Department of Anesthesiology, ${ }^{3}$ Department of Department of Oral \& maxillofacial Surgery, ${ }^{4}$ Department of Cardiothoracic Surgery, Icahn School of Medicine at Mount Sinai, New York, ${ }^{2}$ Department of Anesthesiology, Memorial Sloan Kettering Cancer Center, New York

Address for correspondence: G. Silvay, MD, PhD, Department of Anesthesiology Box 1010, Icahn School of Medicine at Mount Sinai, 1 Gustave L Levy Place, New York, NY 10029.

Phone: +212.241.8346, Fax: +212.426.2009
C. Everett Koop simplified the statement by claiming: "You are not healthy without good oral health" (2).

The association between poor dental hygiene and infection is well recognized. In 1949, Lee wrote that the patient scheduled for surgery had to be seen and assessed by an anesthesiologist with specific concentration on the condition of the respiratory, circulatory, and vascular systems, as well as on that of teeth and gums, along with the state of nutrition, kidney and liver functions, psychological status and previous anesthetic and surgical experiences (3). In fact, these same elements of a preoperative evaluation are still important in todays practice. One of the largest hurdles that still exist is the dental clearance.

The American Heart Association guidelines on infective endocarditis and management of heart valve disease focus on the link to poor dental hygiene. While the recommendations do not specifically address the issue of prophylactic dental evaluation prior to cardiac or major vascular surgery, they emphasize the risk of bacteremia and allude to correlation with poor dentition and daily dental activities (4).

Dental disease is the most common source of infections worldwide (5). Chronic infections such as periodontal disease may play a role in the development of cardiac disease and diabetes mellitus, and are implicated in organ transplant rejection. Periodontitis is a local inflammatory process as a result of bacterial infection of the supporting structures of the teeth and may contribute to the risk of cardiovascular disease. People with a history of root canal therapy were found to be more likely to have coronary artery disease (6).

Currently, dental screening is not a routine practice in many hospitals and cardiothoracic and vascular services. There are several potential reasons for this omission. Firstly, patient education regarding this association may be lacking. Secondly, the surgeons may overlook the importance of eradicating the potential source of infection before cardiac surgery. Thirdly, patients may not have adequate insurance coverage. A study found that $31 \%$ of senior patients in Florida had not seen a dentist in the previous five years (7). These patients may experience a higher incidence of poor oral hygiene, tooth decay, and periodontal disease. Another retrospective study of patients who underwent valvular surgery between 1997 and 2001 identified a high prevalence of poor dental health in valve disease patients, with only $17.3 \%$ of the patients dentally healthy (8). 
185-187

Thus, patients presenting for surgery, who have not had a dental examination for years, may be harboring undiagnosed oral infection that can significantly compromise the surgical outcomes. As a result, too many patients are scheduled for cardiac surgery without dental evaluation. Involvement of the primary physicians and cardiologists may improve the situation. In our institution, patients participating in the Aortic Surveillance Program and Mitral Valve Repair Center are referred for dental care from the time of first encounter. If the operation is indicated, they are getting dental clearance for open heart surgery. For all other patients, after indication for an elective surgery, the dental clearance is requested. In the case of a missing dental evaluation during the first visit in the preoperative evaluation clinic, we are able to arrange the basic dental evaluation in our oral surgical department. If the patient needs invasive dental intervention, the situation is reevaluated and usually the elective surgery is delayed.

Surgeons and dentists often work hand in hand to optimize the oral health of patients prior to cardiac operations. A tooth extraction or treatment of a root canal is not an uncommon procedure prior to surgery. A recent study investigated potential risks that may be associated with the pre-surgical dental operation. In this retrospective study, the authors reviewed the medical records of patients, who had been scheduled for heart surgery and had pre-surgical dental operation. They identified the occurrence of major adverse events following tooth extraction before cardiac surgery and questioned the benefits vs. the risks. The authors alert physicians to evaluate the individualized risk of anesthesia and surgery in this patient population (9). In our letter to the editor, titled "How important is dental clearance for elective open heart operation" (10), we have indicated that the study does not provide evidentiary support for a definitive recommendation for or against dental surgery prior to cardiac operation. We suggest that in case of an urgent or emergency surgery (for example acute coronary syndrome, dissecting aortic aneurysm) the dental evaluation is postponed.

The timing of the dental procedure in relation to the cardiac surgery affects the adverse events $(11,12,13)$. Some authors have questioned the traditional approach of performing dental procedures in advance, advocating that concomitant dental and cardiac surgeries do not alter cardiac outcomes and reduce the anesthetic and infection risks and the costs. (14) Prior to accepting it as an established option, this notion needs to be researched further.

The timely administration of antibiotic prophylaxis with procedure-appropriate antimicrobial selection is another essential aspect of infection control. Coordination and coordination among the health care team members in the preoperative period is critical and can be facilitated by a comprehensive perioperative practice model $(12,13)$.

The American Heart Association guidelines on prevention of infectious endocarditis emphasize the importance of eradicating dental disease, which in our opinion supports the trend for preoperative oral evaluation and treatment in all patients undergoing heart valve operation (15). The Society of Thoracic Surgeons and the American Society of Anesthesiologists do not provide distinct recommendations.
At present, there is no consistent practice or clear guidelines regarding the indications for preoperative dental assessment or oral surgery prior to elective cardiac surgery.

The trends in modern dentistry focus on the maintenance of oral health and prevention of dental illness. There are several venues, at which there is an opportunity for organizing the routine basic dental evaluation of the population, such as at school for students, yearly health evaluations for employees and during military service. The professional involvement and guidance of a dentist is essential for this endeavor (16).

The research articles on the subject include a small number of patients, have a variable scientific level of evidence, and yield contradicting results. Additionally, the outcomes are likely affected by the exact nature of the dental disease; however, the distinction between acute symptomatic dental infection and chronic dental disease is not consistently clarified in the publications. Therefore, developing definite conclusions is challenging and more extensive prospective studies are needed.

In our opinion, the evaluation of oral hygiene in elective surgery with high infection risk, including implantation of prosthetic devices, organ transplantation and oncological procedure, in the setting of immunosuppression, may prevent a dental source of bacteremia and reduce the incidence of endocarditis, perioperative infections such as device-related, wound and systemic infections and therefore improve the outcomes.

The detection of pathological conditions and optimal treatment planning of the affected teeth for patients scheduled to undergo major surgery are of great importance.

\section{References}

1. Miller WD. The human mouth as a focus of infection. Dent Cosmos 1891; 33: 689-713.

2. US Department of Health and Human Services. The oral health of America. The surgeon general: Executive summary, Rockville MD. National Institute of Health 2000.

3. Lee JA: The anaesthetic out-patient clinic. Anaesthesia 1949; 4: 169174.

4. Dajani AS, Taubert KA, Wilson W et al. Prevention of bacterial endocarditis: recommendation by the American Heart Association. JAMA 1997; 277: 1794-1801.

5. Yasny JS, Herlich A. Perioperative dental evaluation. Mt Sinai J Med 2012; 79: 34-45.

6. Gordon SC, Barasch A, Foong WC. Does dental disease hurt your heart? J Can Dent Assoc 2005; 71: 93-95.

7. Gilbert GH, Duncan RP, Crandall L et al. Older Floridians attitudes toward and use of dental care. J Aging Health 1994; 6: 89-110.

8. Wu GH, Manzon S, Badovinac R, Woo SB. Oral health, dental treatment, and cardiac valve surgery outcomes. Spec Care Dentist 2008; 28: 65-72.

9. Smith MM, Barbara DW, Mauermann WJ et al. Morbidity and mortality with dental extraction before cardiac surgery. Ann Thor Surg 2014; 97L: 838-844. 
10. Gandhi N, Silvay G. How important is dental clearance for elective open heart surgery. To the Editor. Ann Thor Surg 2015; 99: 377-381.

11. Yasny JS, Silvay G. The value of optimizing dention before cardiac surgery. J Cardiothor Vasc Anesth 2007; 21: 587-591.

12. Lockhart PB, Brenan MT, Thornhill M, et al. Poor oral hygiene as a risk factor for infective endocarditis-related bacteremia. J Am Dent Assoc 2009; 140: 1238-1244.

13. Flynn BC, Silvay G. Value of specialized preanesthetic clinic for cardiac and major vascular surgery patients. Mount Sinai J Med 2012; 79: 13-24.

14. Lam D, Wright K, Archer B. Is it safe to perform dental and cardiac valve surgeries concomitantly? J Oral Maxillofac Surg 2013; 71: 1000-1004.
15. Wilson W, Taubert KA, Gewitz M et al. A guideline from the American Heart Association Rheumatic Fever, Endocarditis, and Kawasaki Disease Committee, Council on Cardiovascular Disease in the Young, Council on Cardiovascular Surgery and Anesthesia, and the Quality of Care and Outcomes Research interdisciplinary Working Group. Circulation 2007; 11: $1736-1754$.

16. Pavleova G, Vesela S, Stanko P. Prevalence of dental caries in dentistry students. Bratisl Lek Listy 2015; 116: 93-95.

Received June 5, 2015. Accepted August 18, 2015. 